\title{
Surveying ADR knowledge and practices among US gastroenterologists
}

\author{
Michael Peng ${ }^{1}$, Douglas Rex MD, MACG ${ }^{2}$ \\ ${ }^{1}$ Indiana University School of Medicine, ${ }^{2}$ Indiana University School of Medicine, \\ Division of Gastroenterology \& Hepatology
}

\section{Background:}

The adenoma detection rate (ADR) is of primary importance to the quality of screening colonoscopy. An online survey was conducted to assess knowledge and practices on ADR.

\section{Methods:}

Paid questionnaire distributed by email. Eligible respondents were board certified gastroenterologists who perform $>80$ colonoscopies per month with 3 to 35 years after fellowship.

\section{Results:}

$39 \%$ were unaware that ADR applies only to screening colonoscopies and $76 \%$ incorrectly answered that ADR includes sessile serrated polyps/adenomas. $51 \%$ of respondents incorrectly believe the threshold is set at $25 \%$ because $25 \%$ is a national ADR average. Many also believe the threshold depends on the patient population (current evidence suggests adjusting ADR for factors other than age and sex is unnecessary). $75 \%$ ranked ADR as highly important. $80 \%$ reported tracking ADR. A busy practice was the most common reason for not tracking ADR. Caps, chromoendoscopy, and good bowel preparation were viewed as valuable for improving ADR (this is true except for caps). HD colonoscopes and education were considered less valuable (although evidence suggests HD and education are associated with improved ADR). 57\% reported not sharing ADR information with their patients, and $59 \%$ reported no patients in the past 6 months asking for their ADR.

\section{Conclusion:}

The importance of ADR as a validated quality measure is well understood, but there are misconceptions among gastroenterologists regarding the definition and measurement of ADR and which methods are proven to increase ADR. Patients are having very little impact on ADR measurement. 